\title{
USO DE EISENIA FOETIDA (OLIGOQUETOS: LUMBRICIDAE) PARA LA PRODUCCIÓN DE BIOABONO, BOGOTÁ - COLOMBIA
}

\author{
USE OF EISENIA FOETIDA (OLIGOCHAETA: LUMBRICIDAE) \\ FOR COMPOST PRODUCTION, BOGOTÁ - COLOMBIA
}

\author{
${ }^{1}$ Paola Rivera Gallegos, ${ }^{2}$ Andrea Yate-Segura \\ ${ }^{1}$ Ingeniero Ambiental, Universidad Nacional Abierta y a Distancia. ${ }^{2}$ Ingeniero químico, Magíster en Ingeniería, \\ Docente Escuela de Ciencias Agrícolas, Pecuarias y del Medio Ambiente, Universidad Nacional Abierta \\ y a Distancia - UNAD, Investigador semillero de Investigación PIDMA \\ ${ }^{1}$ 1ing.paolarivera17@gmail.com, ${ }^{2}$ andrea.yate@unad.edu.co
}

\section{RESUMEN}

Este documento está enfocado en la generación de Bioabono mediante el uso de la lombriz Eisenia Foetida (Oligoquetos: Lumbricidae), la cual, en condiciones metabólicas adecuadas, fomenta la degradación de residuos sólidos orgánicos. Este proyecto evalúa el proceso de obtención de Bioabono a partir de residuos sólidos orgánicos generados en el barrio Álamos de Bogotá, Colombia, mediante un trabajo en conjunto con la comunidad, para proyectar la cantidad de que se puede producir en este sector, en línea con las políticas nacionales que pretenden introducir la separación y aprovechamiento de residuos desde los hogares. Se logró establecer que los residuos orgánicos representan el $30 \%$ de los residuos generados en el sector, a partir de los cuales se diseñaron tres camas de lombricultivo, con diferentes sustratos, siendo la cama con mayor proporción (60\%) de residuos de yuca y papa la que presentó mayor rendimiento respecto al compost obtenido. El abono obtenido presentó una calidad similar en las tres camas analizadas, evidenciando un exceso de humedad respecto la solicitada en la norma NTC 5167, lo que sugiere que el producto obtenido requiere un proceso de secado antes de su comercialización.

Palabras clave: Compost, Mecanismos de desarrollo limpio, Residuos sólidos orgánicos, Sustrato, y Vermicultura.

\section{ABSTRACT}

This document is focused on the generation of compost through the use of the Eisenia Foetida earthworm (Oligochaetes: Lumbricidae), which, at suitable metabolic conditions, promote the degradation of organic solid waste. This project evaluates the process of obtaining compost from organic solid waste generated in Álamos neighborhood, Bogotá, Colombia, 
through joint work with the community, in order to determine the amount of natural fertilizer that can be produced in the sector, in line with the policies of national organizations that intend to introduce the separation and use of households waste. It was established that organic waste accounts for $30 \%$ of the waste generated in the sector, from which three worm farming beds were designed, with different substrates, the bed with the highest proportion (60\%) of cassava and potato residues being the one that produced the greater yield respect to the compost obtained. Regarding the quality of the product, the results obtained are similar in all the beds, reaching an excess of total nitrogen with respect to that required for commercialization. The results also present excess humidity, which suggests a drying process after obtaining the compost is needed.

Keywords: Vermiculture, Soil Management, Organic Solid Waste, Substrate, Compost and Clean Development Mechanisms.

\section{INTRODUCCIÓN}

De acuerdo con el Informe regional acerca del manejo de los residuos sólidos en América Latina y el Caribe, generado por el Banco Interamericano de Desarrollo en el año 2010, la generación per cápita de Residuos Sólidos Domésticos (RSD) llegó a 0,63kg/hab/día, mientras que la generación de Residuos Sólidos Urbanos (RSU), ascendió a 0,93kg/hab/día, lo que equivale a 295.000 toneladas de RSD y 436.000 de RSU, aproximadamente.

En el caso de Colombia, para el año 2008 se encontró que las ciudades capitales tales como Medellín, Cali, Barranquilla y Bogotá generaron un aproximado de 11.275toneladas/día de residuos sólidos municipales, siendo aproximadamente el $41 \%$ residuos orgánicos (Jaramillo Henao \& Zapata, 2008). En el año 2015, se estimó que Bogotá generó aproximadamente 6.500toneladas de residuos sólidos/día, distribuidos en 20 localidades, siendo la localidad de Engativá después de Kennedy, la que más residuos generó a la ciudad (Alcaldia mayor de Bogotá D.C., 2015). Aunque el manejo, la separación en la fuente y el aprovechamiento de residuos hacen parte de las políticas nacionales, no se cuenta con información actualizada de los residuos generados por las localidades, ni barrios de Bogotá, y los habitantes aún no realizan procesos de separación y aprovechamiento de sus residuos.

En este sentido, el vermicompostaje se convierte en una opción viable por trabajar en las comunidades, ya que es un método de aprovechamiento de residuos orgánicos que consiste en el cultivo intensivo de la lombriz terrestre - la lombriz roja californiana (Eisenia foetida) cuyo sustrato son los residuos orgánicos putrescibles o biodegradables, los cuales una vez transformados, pueden ser aprovechados como abono para cultivos agrícolas (Yate \& Fuquene, 2017). Si bien, en términos generales el manejo de esta Lombriz es muy sencillo, requiere de condiciones ambientales de humedad, temperatura, $\mathrm{pH}$, riego, aireación y alimentación óptima para su desarrollo (Cardenas, 2014; Cajas, 2009; Ferruzzi, 1986). La lombriz Eisenia Foetida es ideal para procesos agroforestales, pues es capaz de sintetizar los desechos orgánicos como estiércoles de los animales y vegetales sobrantes de los cultivos. Adicionalmente, su facilidad de crianza y alta reproducibilidad en condiciones óptimas la hace apropiada para procesos de vermicompostaje (Ferruzzi, 1986). 
El objetivo principal de este estudio fue evaluar el proceso de vermicompostaje realizado a partir de tres sustratos procedentes de residuos sólidos orgánicos putrescibles, generados por una fracción de los habitantes pertenecientes al barrio Álamos, ubicado en la localidad de Engativá. El estudio involucra la identificación de cantidad y composición de los residuos residenciales generados, y la determinación de las posibilidades de comercialización del Bioabono obtenido.

\section{MATERIALES Y MÉTODOS}

La metodología empleada para la generación de Bioabono a partir del uso de Eisenia Foeti$d a$, se basa en un diseño de tipo experimental ejecutado en cinco fases: 1) Encuesta realizada a la comunidad; 2) determinación de la cantidad de residuos generados; 3) caracterización de residuos; 4) Vermicompostaje; 5) evaluación de la calidad del Bioabono.

\section{ZONA DE ESTUDIO}

El proyecto se desarrolló en el barrio Álamos, Localidad Engativá, cerca al aeropuerto el Dorado, en la ciudad de Bogotá. Se encuentra entre las coordenadas $4^{\circ} 42^{\prime} 31^{\prime \prime} \mathrm{N}$ y $74^{\circ} 07^{\prime} 11^{\prime \prime} \mathrm{W}$, con una precipitación entre 500 y $1000 \mathrm{~mm}, \mathrm{y}$ una temperatura promedio entre $9^{\circ} \mathrm{C}$ y $14^{\circ} \mathrm{C}$ (Murcia, 2012). El proceso de vermicompostaje se llevó a cabo dentro de una construcción totalmente protegida contra los efectos del clima o animales, y con cubierta para garantizar un proceso continuo de condensación, de tal forma que los residuos siempre estén humedecidos.

\section{CIUDADANÍA}

Para el desarrollo de este proyecto, fue necesario aplicar encuestas aleatorias a la comunidad. Estas contaron con su debido consentimiento informado e incluyeron preguntas cerradas de respuesta afirmativa o negativa y preguntas abiertas de respuesta corta, las cuales se presentan a continuación:
- ¿Sabe usted qué son los residuos orgánicos?

- ¿Conoce usted cómo se deben separar los residuos sólidos?

- ¿Actualmente, usted consume frutas, verduras y legumbres en su hogar?

- ¿Aproximadamente cuántas libras de frutas, verduras o legumbres consume durante una semana?

- ¿Qué uso le da usted a los residuos orgánicos de su hogar?

- ¿Sabe usted qué es el Bioabono?

- ¿Tiene conocimiento acerca de los planes de agricultura urbana que actualmente existen en la localidad de Engativá?

- ¿Le gustaría participar de un proyecto en el cual se le da una disposición diferente al relleno sanitario a los residuos orgánicos generados en su hogar?

- Por último, díganos qué cantidad de habitantes hay en su casa o apartamento.

Las encuestas fueron realizadas únicamente a mayores de edad, debido a su potencial interés en el tema y que, en caso de iniciar procesos de separación y recolección in situ de los residuos orgánicos generados, podrían involucrar a su núcleo familiar y tener una influencia positiva en ellos.

\section{RESIDUOS GENERADOS}

La composición de los residuos se realizó en el primer mes del proyecto empleando el método de cuarteo, el cual consistió en seleccionar aleatoriamente tres bolsas de basura de $5 \mathrm{~kg}$ cada una, y depositar los residuos encima de un plástico para poder identificar los residuos que estaban presentes en dicha muestra, este proceso se realizó por triplicado.

\section{VERMICOMPOSTAJE}

Se clasificaron los residuos orgánicos putrescibles aptos para la generación de Bioabono. Posteriormente se determinaron los pre-tratamientos requeridos, tales como, reducción de 
tamaño de partícula y premezclado de componentes según sus propiedades ácidas con el fin de reducir posibles problemas de acidez en el suelo. Después, se diseña la cama en un tanque de polietileno, de 50 galones de capacidad; inicialmente se emplea una capa de tierra negra sin abono de aproximadamente $20 \mathrm{~cm}$, a continuación, se coloca, como pie de cría en las camas, una capa inicial de lombrices entre 10 y $15 \mathrm{~cm}$ aproximadamente, una capa delgada del sustrato de aproximadamente $4 \mathrm{~cm}$; y, por último, una segunda capa de tierra de $10 \mathrm{~cm}$ y se humedece el sustrato.

Para generar el efecto invernadero requerido se tapa el Bioabono con un plástico de color negro, lo cual contribuye a mantener las condiciones de humedad del sustrato y las condiciones fisicoquímicas del suelo.

La frecuencia de alimentación de las lombrices es cada 8 días, en los que igualmente se realiza un volteo del material, revisando la humedad de la tierra de forma casera, a través del método de las 10 gotas de agua; además, se mide la temperatura empleando un termómetro digital de punzón para alimentos 0131 capaz de controlar $-50^{\circ} \mathrm{C}+300^{\circ} \mathrm{C}$, con un margen de error de $\pm 1^{\circ} \mathrm{C} /{ }^{\circ} \mathrm{F}$. El pH se mide empleando tiras indicadoras marca Macherey Nagel, capaces de indicar el $\mathrm{pH}$ en un rango de 0-14 unidades, con lo cual se verifica el estado de maduración del Bioabono. La revisión y volteo del lombricultivo se realiza con una frecuencia de aproximadamente dos meses.

Comportamiento poblacional: para calcular el crecimiento poblacional de cada una de las camas diseñadas, se sigue la ecuación 1 , a fin de establecer si los residuos empleados afectan de alguna forma el crecimiento de las lombrices.

$$
P(t)=P\left(t_{0}\right) \times 2^{2 / 90} \quad E c .1
$$

Donde:

$\mathrm{P}(\mathrm{t})=$ Población total

$\mathrm{P}(\mathrm{t0})=$ Población inicial

t/90 = tiempo empleado en días sobre la pro-

ducción de nuevas lombrices.

La ecuación 2 permite establecer el promedio de lombrices presentes en el lombricultivo

$\mathrm{kg}$ humus $*\left(\frac{\text { Número de lombrices }}{1 \mathrm{~kg} \text { humus }}\right)=$ Promedio de lombrices $/ \mathrm{kg}$ EC. 2

Rendimiento del lombricultivo: se determina mediante un balance másico siguiendo la ecuación 3.

$\%$ rendimiento del lombricultivo $=\left(\frac{\text { Producto final } \mathrm{kg}}{\text { materia prima inicial kg }} * 100\right)$ Ec. 3

\section{RECOLECCIÓN DEL BIOABONO}

Visualmente se evidencia la formación de Bioabono en las camas, por lo que estas se cubren con una lona sobre el sustrato y se colocan más residuos orgánicos putrescibles. De esta forma se logra que las lombrices suban hasta ese nivel, facilitando la recolección de estas, y permitiendo extraer el Bioabono, sin que este contenga lombrices en su interior. Finalmente, el Bioabono se recoge, se retiran las partes sólidas y se tamizan las muestras para su posterior caracterización.

\section{EVALUACIÓN DEL BIOABONO}

Durante esta fase se realiza una serie de análisis fisicoquímicos al Bioabono para contrastarlos con la Norma Técnica Colombiana NTC 5167 de 2004, establecida por el ICA, para la comercialización de Bioabono en el Territorio Nacional (ICONTEC, 2004).

\section{MÉTODOS ANALÍTICOS}

Para determinar la calidad del Bioabono, fue necesario realizar la medición de los siguientes análisis: 
- pH: la medición del pH se realizó empleando un potenciómetro HANNA Instruments, medidor de $\mathrm{pH}$, empleando buffer de 4 y 7 , siguiendo el procedimiento descrito en el método ASTM D4972-01 (2007).

- Contenido de humedad: el contenido de humedad del abono se midió en un horno marca Furnace 48000 (Barnstead International, Estados Unidos), capaz de controlar $\pm 0,1^{\circ} \mathrm{C}$, de acuerdo con el procedimiento establecido en el Standar method ASTMD 2216-10.

- Temperatura: la temperatura del abono en el laboratorio se midió, con un equipo multiparámetro marca Hanna Instruments 211 (Estados Unidos), con un nivel de precisión de $\pm 1^{\circ} \mathrm{C}$.

- Densidad Aparente: el contenido de densidad aparente del abono se calculó siguiendo el método de excavación definido por (García, 2011), siguiendo la ecuación 4.

$$
\text { Dap }=\frac{\text { Peso de suelo seco }(\text { gramos })}{\text { Volumen total }\left(\mathrm{cm}^{3}\right)} \quad \text { Ec. } 4
$$

- Conductividad Eléctrica: la conductividad eléctrica se determinó empleando un equipo multiparámetro marca Hanna Instruments 211 (Estados Unidos) con un nivel de precisión de $\pm 1\left(20^{\circ}\right)$, siguiendo el procedimiento descrito en la norma NOM-021-RECNAT-2000, para determinación de conductividad eléctrica en suelos.

- Determinación de textura por sedimentación: se realiza siguiendo el procedimiento establecido por (García, 2011) empleando un diagrama triangular de suelos.

- Determinación de Nitrógeno Total: el método empleado fue SM4500NorgC SM4500NH3-C, realizado por el laboratorio CIAN LTDA.

\section{RESULTADOS Y DISCUSIÓN}

\section{RESIDUOS GENERADOS}

La Figura 1 presenta los resultados promedio obtenidos a partir del método de cuarteo, donde se establece que el porcentaje más alto de residuos en general corresponde a los plásticos, contaminados o no contaminados, alcanzando un $45 \%$. Seguido de estos se encuentran los residuos orgánicos con un $30 \%$, los residuos de papel sanitario con un $12 \%$ y finalmente un $13 \%$ de los residuos de papel que involucran periódicos y revistas.

Entre los principales residuos orgánicos putrescibles que se identificaron fueron residuos de frutas, tales como, guayaba (Psidium guajava), naranja (Citrus aurantium L.), feijoa (Acca sellowiana), tomate de árbol (Solanum betaceum) y maracuyá (passiflora edulis). También se encontraron residuos de verduras, cáscaras de papa (Solanum tuberosum), cáscaras de plátano (Musa balbisiana), aguacate (persea americana), tomate (solanum lycopersicum), entre otros.

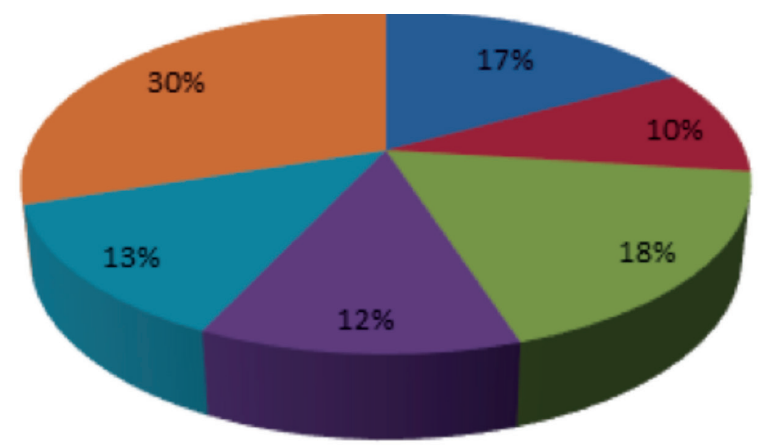

\author{
- Plásticos contaminados \\ - Bolsas plásticas \\ - Botellas plásticas \\ - Papel sanitario \\ - Otros papeles \\ nesiduos orgánicos
}

Figura 1. Residuos domiciliarios.

Fuente: Autores 


\section{LOMBRICULTIVO}

Posterior a la siembra de las lombrices, y considerando la cantidad de residuos de cáscara de naranja generados en el sector, se adicionaron a la cama $6 \mathrm{~kg}$ de estas para su descomposición. Sin embargo, los ácidos presentes en la descomposición de la naranja, hacen que el $\mathrm{pH}$ se aumente a 9,5 unidades, generando un exceso de lixiviados, lo cual altera el porcentaje de humedad y hace que la mayoría de lombrices mueran, quedando 12 lombrices con las que se realiza nuevamente inoculación, utilizando tierra negra, y cáscaras de papa y de yuca como sustrato, con el fin de garantizar un medio de cultivo neutro, y sin la presencia de ácidos o de lixiviados.

De acuerdo a lo indicado por Taco (2010), las sustancias ácidas como la naranja y el limón producen una liberación de bases y un alto contenido de amoniaco debido a la descomposición natural de los ácidos orgánicos, lo que aumenta el pH. En este sentido, es posible resaltar que los residuos cítricos, como los de la pulpa de naranja, en altas proporciones, cuentan con un $\mathrm{pH}$ inicial de 2 a 2.5 unidades, que no permite el desarrollo de las lombrices sino hasta 2 o 3 semanas después de su adaptación al medio, momento en que el $\mathrm{pH}$ es naturalmente regulado. Desde un enfoque práctico, para el uso de estas sustancias en vermicompostaje se aconseja realizar un pre-compost antes de agregar el material al espacio donde están ubicadas las lombrices (Taco, 2010).

Para la creación del pie de cría - cama 1, $60 \%$ de residuos de papa y yuca y $40 \%$ de residuos de verduras, tales como; arveja (Pisum sativum), zanahoria (Daucus carota), tomate (solanum lycopersicum), y espinaca (Spinacia oleracea) se usaron como sustrato. Con este sustrato hay un aumento en la población de lombrices, lo que permite generar dos camas adicionales; la cama 2, tiene como sustrato un $20 \%$ de residuos de frutas (como tomate de árbol (Solanum betaceum), papaya (Ciraca papaya), guayaba (Psidium guajava), banano (Musa balbisiana), mango (Mangifera indica)), $40 \%$ residuos de verduras, y $40 \%$ de residuos de papa (Solanum tuberosum); mientras que la cama 3 tuvo como sustrato $100 \%$ de cáscaras de plátano (Musa balbisiana), dichos residuos se emplearon debido a que. según el cuarteo, son los más generados en el sector. En las Figuras 2 y 3 se presentan las camas.

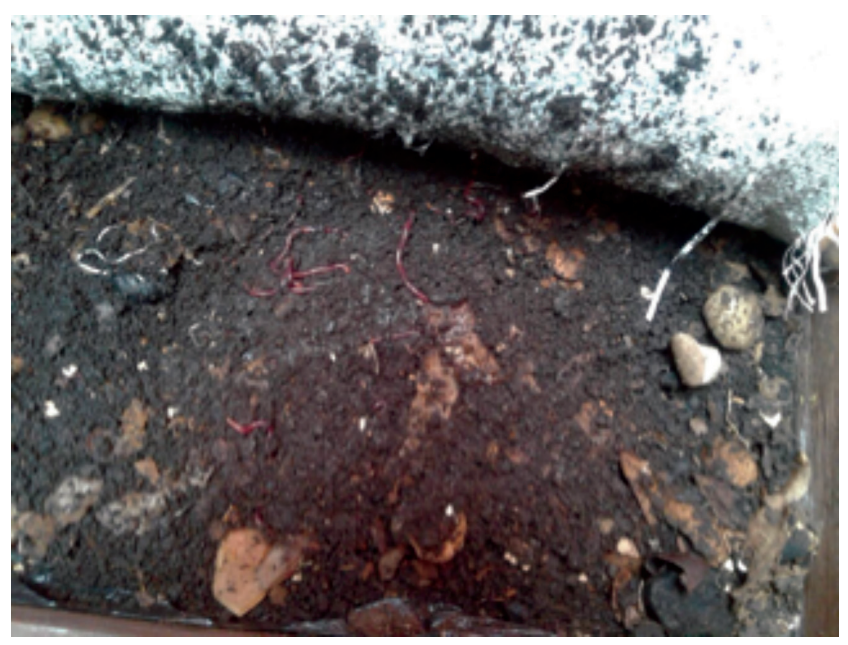

Figura 2. Pie de cría-Cama 1. Fuente de: Autores

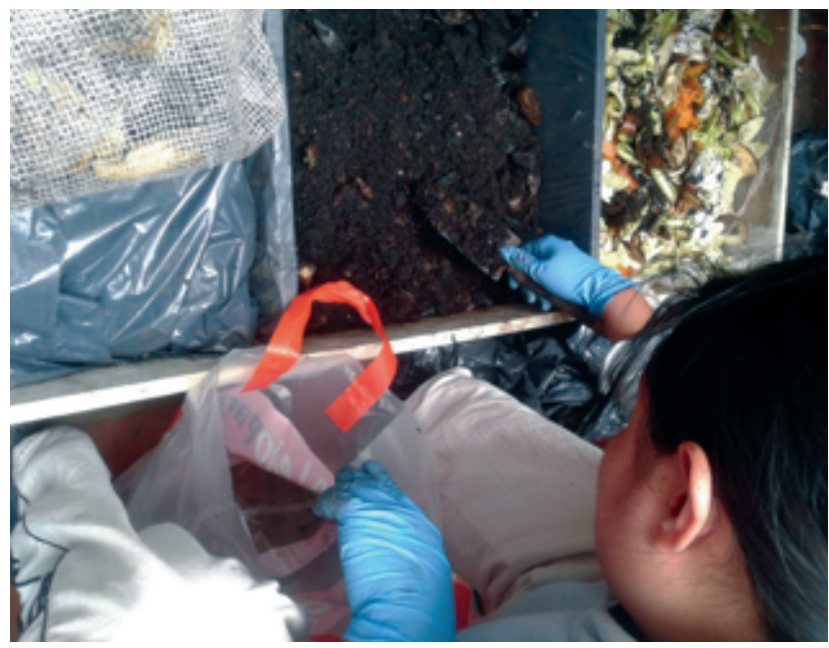

Figura 3. Camas 2 y 3.

Fuente de: Autores 


\section{CALIDAD DEL BIOABONO}

En la Tabla 1 se presentan los resultados de calidad del abono junto con los parámetros establecidos en la norma NTC 5167 del 2004 que establece las condiciones fisicoquímicas que debe tener un Bioabono según el ICA, para poder ser comercializado. En esta se observa que las tres camas generadas presentan $\mathrm{pH}$ neutros, mientras que la humedad reportada en todas las camas es superior a la requerida en la norma. Es decir que es necesario generar un proceso de secado previamente para su comercialización.

La densidad aparente refleja la porosidad total en un suelo y es importante para el manejo de estos mismos; además, la densidad aparente refleja la compactación y facilidad de circulación de agua y aire. Es decir, cuanto mayor sea la densidad, menor el espacio poroso para el movimiento del agua, crecimiento y penetración de raíces, y el desarrollo de las plantas (Observatorio Nacional de la Degradación de Tierras y Desertificación, 2014); De acuerdo con esta información se concluye que la densidad aparente de las camas 1 y 3 , es la adecuada ya que los valores se encuentran dentro de los rangos establecidos.
Por otra parte, en la cama 2 se supera el valor requerido debido, principalmente, a la presencia de frutas con alto contenido de humedad, lo cual altera el nivel de porosidad del suelo. Si bien la conductividad eléctrica no cuenta con un valor específico en la norma, es importante, considerando que esta permite establecer si el abono obtenido es salino o no, y las implicaciones que tendría en la siembra.

Al igual que la conductividad eléctrica, la norma solicita que valores por encima de $1 \%$ del nitrógeno total sean reportados. En este caso, todas las camas superan este valor debido a la presencia de fluorescencia en la clorofila, como resultado de la interacción ozono- nitrógeno. (Sánchez, 2014)

Finalmente, es posible establecer que la cama 1 es la que presenta mayor rendimiento del lombricultivo; por su parte, la cama 3 también presenta un alto porcentaje de rendimiento del lombricultivo, debido a la alta concentración de glucosa presente en las cáscaras de plátano y banano. 
Tabla 1. Resultados de laboratorio y comparación con la norma

\begin{tabular}{|c|c|c|c|c|c|}
\hline Parámetro & Unidad & Cama 1 & Cama 2 & Cama 3 & NTC 5167 \\
\hline $\mathrm{PH}$ & Unidades & $7,18 \pm 0,06$ & $7,37 \pm 0,02$ & $7,51 \pm 0,05$ & $4,0-9,0$ \\
\hline Temperatura & ${ }^{\circ} \mathrm{C}$ & 20,00 & 20,00 & 20,00 & - \\
\hline Humedad & $\%$ & $64,5 \pm 6,56$ & $78,8 \pm 2,02$ & $54,5 \pm 2,02$ & $35 \% \operatorname{Max}$ \\
\hline Textura & - & $\begin{array}{l}\text { Franco- } \\
\text { arenoso }\end{array}$ & Franco-arenoso & Franco-arenoso & - \\
\hline Densidad & $\mathrm{gr} / \mathrm{cm}^{3}$ & 0,60 & 0,79 & 0,54 & 0,6 \\
\hline C.E & milimhos/cm & 2,77 & 3,43 & 2,77 & Reportar \\
\hline Nitrógeno Total ${ }^{1}$ & $\%$ & 2,60 & 2,00 & 1,80 & Reportar si > $1 \%$ \\
\hline Materia prima & $\mathrm{Kg}$ & 0,2 & 2,0 & 2,0 & - \\
\hline Producto final & $\mathrm{Kg}$ & 8 & 6,64 & 11,68 & - \\
\hline $\begin{array}{l}\text { Rendimiento } \\
\text { del lombricultivo }\end{array}$ & $\% /$ mes & $285 \%$ & $30.18 \%$ & $53.09 \%$ & - \\
\hline
\end{tabular}

NTC 5167 = Norma Técnica Colombiana 5167

La Tabla 2 presenta los resultados del comportamiento poblacional de las lombrices para las tres camas trabajadas después de un año de estudio. A partir de esta se concluye que el pie de cría inicial, después de ser estabilizado empleando residuos con alto contenido de glucosa, tuvo un aumento poblacional de 12 lombrices a 304 lombrices, aproximadamente. También demuestra que las condiciones físicas en las cuales están ubicadas las lombrices son las adecuadas, por ello se facilita su desarroIlo. Con respecto a la cama 2 y 3 , estas solo alcanzaron la mitad del aumento poblacional de la cama 1, lo cual es esperado dado que, aunque las lombrices ya estaban inoculadas y estaban preparadas para otro tipo de sustrato, su aceptación al medio no es tan alta como con la glucosa.

Tabla 2. Comportamiento poblacional del lombricultivo

\begin{tabular}{l|l|l|l|l} 
Cama & Población inicial & \multicolumn{1}{c}{ Población Total } & $\begin{array}{c}\text { Promedio } \\
\text { Iombrices / kg/ } \\
\text { mes }\end{array}$ & $\begin{array}{c}\text { Incremento } \\
\text { poblacional }\end{array}$ \\
\hline Cama 1. & 12 & 304 & 33,71 & 26,33 veces \\
\hline Cama 2. & 20 & 254 & 43,09 & 12,70 veces \\
\hline Cama 3. & 35 & 444 & 75,36 & 12,68 veces \\
\hline
\end{tabular}

\footnotetext{
${ }^{1}$ Según reporte de resultados del laboratorio CIAN LTDA, No. 000803.
} 


\section{CONCLUSIONES}

Aproximadamente el $30 \%$ de los residuos que se generan al interior de un hogar corresponde a residuos de origen orgánico putrescible. En el barrio Álamos, en promedio se pueden generar $355 \mathrm{~kg}$ de estos residuos sólidos, de los cuales se pueden aprovechar aproximadamente $106,5 \mathrm{~kg}$, que corresponden a residuos sólidos orgánicos putrescibles. Lo anterior, sin tener en cuenta que los residuos de plásticos y de papel pueden llegar igualmente a ser llevados a procesos de reciclaje.

Si bien este trabajo evaluó tres sustratos diferentes, incorporando residuos de frutas y verduras, es posible establecer la afinidad de las lombrices rojas californianas a la glucosa, alcanzando rendimientos máximos de $285 \%$ mensual para la cama 1 que contenía un $60 \%$ de residuos de papa y yuca. Esta afinidad se refleja igualmente en el crecimiento poblacional encontrado.

Esta investigación también permite establecer la importancia del tipo de sustrato, dado que las camas 2 y 3 presentan un rendimiento considerablemente bajo, 31,08\% y 53,09\%, respectivamente, con relación a la cama 1 . Lo que sugiere que los sustratos empleados afectan directamente el rendimiento del cultivo y el crecimiento poblacional.

Por otro lado, aunque la caracterización presentó algunas variaciones en cuanto al contenido de nitrógeno total y la humedad, son parámetros que pueden ser mejorados con tratamientos previos a su comercialización.

Finalmente, es posible concluir que este tipo de iniciativas requieren de la participación activa de la comunidad, no solo porque es imperante manejar adecuadamente la separación en la fuente, sino que además de desear comercializar el abono son necesarios altos volúmenes de residuos, lo cual requeriría de la participación de por lo menos el $50 \%$ de los habitantes de Álamos.

\section{AGRADECIMIENTOS}

Queremos hacer un reconocimiento especial a la comunidad del barrio Álamos por su contribución sustancial con el trabajo presentado.

\section{LITERATURA CITADA}

Alcaldia mayor de Bogotá D.C. (2015). Secretaria general de la Alcaldia Mayor de Bogotá D.C. Recuperado de http://www.alcaldiabogota.gov.co/ sisjur/normas/Norma1.jsp?i=62903

Cajas, S. (2009). Efecto de la utilización de aserrin en combinación con bovino, como sustrato en la producción de humus de lombriz eisenia foetida. Escuela superior politécnica de Chimborazo, Riobamba, Ecuador.

Cardenas, B. E. (2014). Guia técnica para el aprovechamiento de residuos orgánicos, a través de metodologías de compostaje y lombricultura. Universidad Nacional de Colombia y Alcaldía Mayor de Bogotá Bogotá, Colombia.

Ferruzzi, C. (1986). Manual de lombricultura. España, Mundi:Prensa.

García, I. A. (2011). Determinación de textura por sedimentación. En Manual del prácticas de Edafología (págs. 28-31). Chiapas, México.

ICONTEC. (2004). NTC 5167 de 2004. Productos para la insdustria agrícola, productos orgánicos usados como abono, o fertilizantes y enmiendas de suelos. Bogotá, Colombia.

Jaramillo H., G., \& Zapata, L. (2008). Aprovechamiento de los residuos orgánicos en Colombia. Universida de Antioquia. Recuperado de http:// bibliotecadigital.udea.edu.co/dspace/bitstream/10495/45/1/AprovechamientoRSOUenColombia.pdf

Murcia, J. F. (2012). Escenarios de cambio climático. Revista técnica IDEAM, 18-30.

Observatorio Nacional de la Degradación de Tierras y Desertificación. (2014). Recuperado de http://www.desertificacion.gob.ar/indicadores/ suelo-da/ 
Sánchez, A. M. (2014). Efectos del ozono y su interacción con el Nitrogeno en seis variedades de hortalizas. Universidad de Valencia, Facultad de ciencias biológicas, 1-73.

Taco, J. C. (2010). Análisis de lombricompuestos a partir de diferentes sustratos. Codazzi, Cesar.
Yate, A., \& Fuquene, D. (2017). Vermicompostaje en el manejo de los residuos sólidos urbanos. Working paper, Recuperado de http://hemeroteca.unad.edu.co/index.php/workpaper/article/ view/1816/2023
Conflicto de Intereses Los autores declaran no tener ningún conflicto de intereses 